\section{Cyclotorsional eye movements during a simulated PRK procedure}

AM Fea, L Sciandra, F Annetta, M Musso, $M$ Dal Vecchio and FM Grignolo
Clinica Oculistica, Universita' di Torino, Dipartimento di Fisiopatologia Clinica, V Juvarra 19, Torino, Italy

Correspondence: AM Fea, Clinica Oculistica di Torino, $\checkmark$ Juvarra 19 10100 Torino, Italy Tel: + 0039011 5666048; Fax: + 0039011539024 E-mail: antoniofea@ interfree.it

Received: 26 September 2004

Accepted in revised form: 25 May 2005

Published online: 1 July 2005

Presented in part at the Winter ECRS Meeting, Munich, 6-10 September 2003

None of the authors have any financial interest on the subject of the paper

\begin{abstract}
Purpose Eye alignment is a serious concern when performing corneal surface ablation. Although several excimer lasers monitor horizontal and vertical movements, little is known about the potential impact of cyclotorsional movements. Dynamic cyclotorsions have been measured on $\mathbf{1 0}$ emmetropic subjects during a simulated PRK treatment.

Methods Cyclotorsional eye movements were measured using a 3D videoculographic system (SMI) in 10 emmetropic subjects in upright and supine position. The subjects were wearing an eyelid speculum and were asked to fixate on a $1^{\circ}$ red spot. The fixation target was either in focus or optically degraded by electronic filters. Data were collected at the University Eye Clinic, Torino, Italy, and the different setting measurements were compared using Student's $t$-test for paired data.

Results Cyclotorsions were significantly higher when subjects were fixating an optically degraded stimulus (upright: $P=0.04$; supine: $P=0.0002)$. The cyclotorsional movements ranged from -13 to $17^{\circ}$. A significant difference in eye orientation was present when changing from the upright to the supine position $(P=0.03)$. Even when correcting for this positional error, significantly higher cyclotorsional movements were detected in the supine position (in focus: $P=0.0043$; optically degraded: $P=0.0008)$. The torsional range was from -14.85 to $14.55^{\circ}$.

Conclusions The high range of cyclotorsional eye movements during a simulated PRK procedure suggests that $3 \mathrm{D}$ tracking could improve surface ablation results when treating high astigmatism or when wavefront guided.

Eye (2006) 20, 764-768. doi:10.1038/sj.eye.6701994; published online 1 July 2005
\end{abstract}

Keywords: cyclotorsional movements; excimer laser; videoculography

\section{Introduction}

Recently, the development of diagnostic and surgical tools has prompted the search for perfect vision. Aberrometry-guided laser treatments are more commonly being performed but require a very accurate and precise placement of the laser spots. The theoretical importance of torsional misalignments can be understood if we consider that a $10^{\circ}$ alignment error results in a $30 \%$ astigmatic, 50\% trefoil and $65 \%$ tetrafoil undercorrection. ${ }^{1}$ It has been previously reported that eye orientation significantly changes when moving from an upright to a supine position ${ }^{2-4}$ and it has been postulated that this might cause errors in astigmatic correction. Relatively little is known about dynamic torsional eye movements, although torsional eye movements during fixation are believed to be significantly higher than orthogonal eye movements. ${ }^{5}$ Cyclotorsional eye movements can be dynamically monitored by means of infrared video-oculography. This method compares favorably with more complex and invasive techniques. ${ }^{6,7}$ During refractive surgery, patients with refractive errors are asked to stare at a fixation light at a close distance, which is usually perceived as focused or moderately defocused even when large refractive errors are present. After the corneal epithelium removal (PRK, LASEK) or lifting of the flap (LASIK) and increasingly during the ablation procedure, the fixation light is perceived as optically degraded and fixation is poorer. To simulate a refractive procedure, emmetropic subjects were asked to fixate on a red spot $\left(1^{\circ}\right)$ presented on a TV monitor at $2 \mathrm{~m}$ distance, which was either in focus or optically degraded. Furthermore, we determined 
whether changing from a sitting to a supine position could not only change the eye orientation as previously reported $^{2-4}$ but might also have some impact on dynamic cyclotorsion.

\section{Materials and methods}

Data were collected under an Institutional Review Board approved protocol at the University Eye Clinic in Torino, Italy. In total, 10 eyes of 10 young (mean age: $24.2 \pm 2$ years) emmetropic (cycloplegic refraction: $+1.00 \mathrm{D}$ to $-0.5 \mathrm{D}$ spherical equivalent) subjects with no known abnormalities of their visual system were included in this study. Strabismus, phorias and any other eye disease were excluded by a complete eye exam including biomicroscopy, ophthalmoscopy, autorefractometry, cover test with prisms, assessment of ocular motility, determination of stereopsis with the Random dot $\mathrm{E}$ and the Lang tests.

Informed consent was obtained from all the participants.

Four different experimental settings were used to assess the cyclotorsional eye movements: the subjects were fixating in upright position (1) a focused stimulus or (2) an optically degraded stimulus, and in supine position (3) a focused or (4) an optically degraded stimulus. The duration of each setting was $30 \mathrm{~s}$. The order of the different experimental settings was pseudorandom. During fixation in the supine position, the stimulus was observed through a mirror system.

The red fixation circular target $\left(1^{\circ}\right.$ at $\left.2 \mathrm{~m}\right)$ was generated by the Cambridge Research System frameware using the Psycho program and presented on a highresolution monitor (Mitsubishi Diamond Scan 17HX). The same stimulus was optically degraded by means of different electronic filters to simulate the perception of the fixation light when the epithelium is removed in PRK or the flap is lifted in LASIK (Figure 1).

Cyclotorsional eye movements were measured using a $3 \mathrm{D}$ videoculography (SMI). The system works with two infrared cameras and calculates the three-dimensional eye position in a real time at a frame rate of $50 \mathrm{~Hz}$. The cameras are mounted in a custom-made rigid frame (Figure 2) that contains two infrared reflecting mirrors
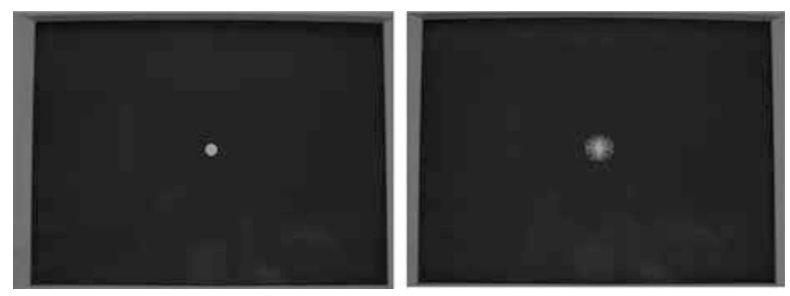

Figure 1 Focused and optically degraded stimuli.

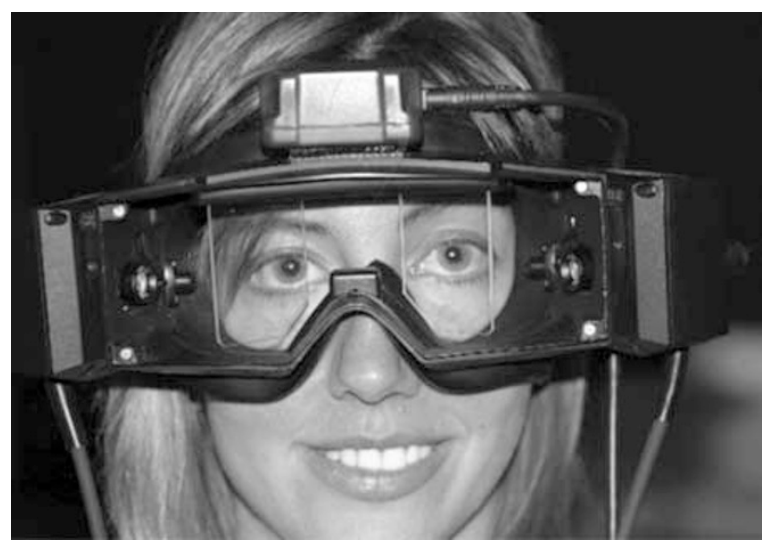

Figure 2 Subject with the 3D videoculography mask.

and three infrared light-emitting diodes for each eye. Spatial resolution is within $0.1^{\circ}$ and the measurement range is -18 (excyclotorsion) to $+18^{\circ}$ (incyclotorsion). When cyclotorsion exceeds $\pm 18^{\circ}$, the measurement is recorded out of range. Vertical and horizontal eye positions were calibrated for each eye using fixation positions over a $20^{\circ}$ range and in $5^{\circ}$ increments along the horizontal and vertical meridians. Torsion was calculated with respect to a reference position defined as zero. The zero point was the average orientation for the lines of sight while fixating a $2 \mathrm{~m}$ distant target over a period of $5 \mathrm{~s}$. The SMI apparatus allows to simultaneously measure the horizontal and vertical eye movements as well as the head rotation. Care was taken as to keep the head in the same position during the experimental setting. Eye torsions that corresponded to head rotations higher than $1^{\circ}$ were discarded. Data were analyzed offline. The SMI program provides a quality index that relates to each frame. Low-quality index frames $(\leqslant 0.7)$ were excluded from the analysis. All the measurements were monocular and the fixating eye was kept open using an eyelid speculum. Topical anesthesia with Oxybuprocaine $0.4 \%$ was used. For each subject one eye was randomly chosen. Hyaluronic acid $0.2 \%$ was used between the experimental settings or when the subjects were complaining of burning or blurring.

\section{Statistical analysis}

In the analysis, because excyclotorsions are reported as negative and incyclotorsions as positive and averaging the values would have underestimated the torsional movements, absolute values were used. The average cyclorotation in the four experimental settings was analyzed. Owing to the eye tracker's time frame, the $\mathrm{z}$ eye position of each subject was determined every $0.02 \mathrm{~s}$. The average standard deviation of each subject's 
torsional movements during the $30 \mathrm{~s}$ recording can thus be used as an index of position instability during each setting. The different setting measurements were compared using a Student's $t$-test for paired data. The average duration of cyclotorsional movements was calculated.

\section{Results}

When moving from an upright to a supine position, the average change in cyclotorsion is $3.3^{\circ}$. This is significantly different from a null hypothesis $(P=2.1$; $t=0.03)$. The average cyclorotation was significantly higher when the subjects were fixating the optically degraded stimulus in the upright position $\left(0.8\right.$ vs $\left.1.33^{\circ}\right)$ and even more in the supine position (1.86 vs $3.45^{\circ}$ ) (Table 1). The subjects had significantly higher dynamic cyclotorsions when in the supine position (focused: $0.8 \mathrm{vs}$ 1.86; optically degraded: 1.37 vs 3.45) (Table 1). The average standard deviation of torsional movements proved significantly higher in the supine position and when the subjects were fixating an optically degraded stimulus (Table 2). The cyclotorsions ranged from -10.5 to +18 diopters (upright focused: from -10.5 to +18 ; upright optically degraded: from -13.1 to +17 ; supine focused: from -12.3 to +17 ; supine optically degraded: from -14.9 to +14.55 ). To further investigate the impact of cyclotorsional eye movements on corneal ablation, we analyzed the duration of cyclotorsional movements. Figure 3 reports the average duration of cyclotorsional eye movements when the subjects were fixating an optically degraded stimulus in the supine position. The

Table 1 Cyclorotation during fixation of focused and optically degraded stimuli in upright and supine position

\begin{tabular}{lcccc}
\hline & $\begin{array}{c}\text { Focused } \\
\text { (mean } \pm \text { stdev) }\end{array}$ & $\begin{array}{c}\text { Optically degraded } \\
\text { (mean } \pm \text { stdev) }\end{array}$ & $T$ & $P$ \\
\hline Upright & $0.8 \pm 0.64$ & $1.33 \pm 1.3$ & 2.36 & 0.04 \\
Supine & $1.86 \pm 1.23$ & $3.45 \pm 1.69$ & 6.07 & 0.0002 \\
$T$ & 3.84 & 4.97 & & \\
$P$ & 0.004 & 0.0008 & & \\
\hline
\end{tabular}

Table 2 The average standard deviation of torsional movements

\begin{tabular}{lcccc}
\hline & $\begin{array}{c}\text { Upright } \\
\text { (mean } \pm \text { stdev) }\end{array}$ & $\begin{array}{c}\text { Supine } \\
\text { (mean } \pm \text { stdev) }\end{array}$ & $T$ & $P$ \\
\hline Focused & $1.45 \pm 1.08$ & $4.01 \pm 1.63$ & 6.81 & 0.00008 \\
Optically degraded & $2.89 \pm 1.35$ & $5.91 \pm 1.82$ & 10.2 & 0.000003 \\
$T$ & 5.21 & 16.88 & & \\
$P$ & $<0.0006$ & $<0.0001$ & & \\
\hline
\end{tabular}

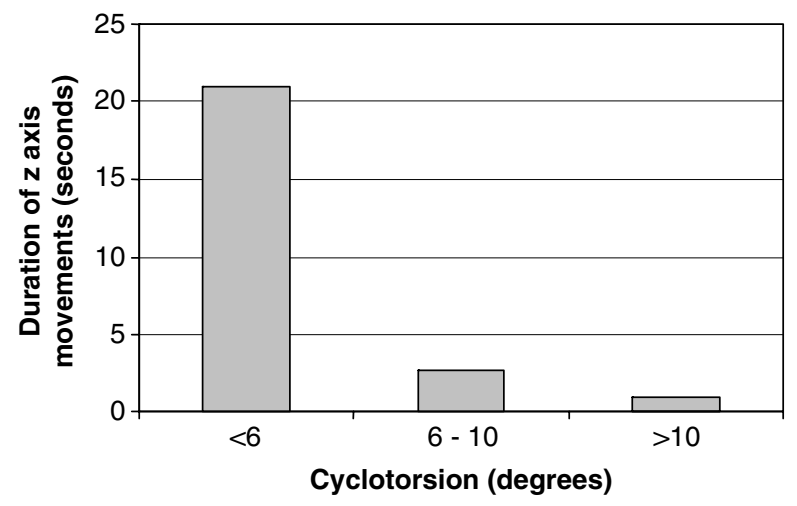

Figure 3 Average duration of cyclotorsional eye movements when patients were fixating an optically degraded stimulus in the supine position.

Table 3 Number of subjects with cyclorotation exceeding $5^{\circ}$

\begin{tabular}{lcc}
\hline & $\begin{array}{c}\text { Upright } \\
\text { (longer than 1s) }\end{array}$ & $\begin{array}{c}\text { Supine } \\
\text { (longer than 1s) }\end{array}$ \\
\hline Focused & $4(1)$ & $8(4)$ \\
Optically degraded & $6(4)$ & $10(6)$ \\
\hline
\end{tabular}

number of subjects who had cyclorotation exceeding $5^{\circ}$ is reported in Table 3. The number of subjects in whom the $>5^{\circ}$ rotation lasted longer than $1 \mathrm{~s}$ is given in brackets. In only one subject, three out-of-range measures $\left(>18^{\circ}\right)$ were recorded.

\section{Discussion}

Previous studies suggested that eye torsion can significantly influence surface ablation due to the change of eye position from an upright to a supine position. ${ }^{2-4}$ The stability of fixation on the horizontal and vertical plane significantly worsens through increased amounts of blur, ${ }^{8}$ and during surface ablation the blurring of the fixation target can increase horizontal and vertical eye movements. To our knowledge, this is the first study that addresses the question of dynamic changes of eye torsion during a simulated surface ablation. Recent papers in fact reported on the difference of eye position during actual refractive surgery ${ }^{4}$ or concentrated on dynamic differences of cyclorotation in the upright $v s$ supine position. ${ }^{8}$ Our experiments demonstrate not only that the $\mathrm{z}$ eye position changes from the sitting to the supine position but also that the dynamic cyclotorsions are significantly higher in the supine position. Furthermore, both the mean cyclotorsion and the mean variability (standard deviation) of cyclotorsional eye movements 
proved significantly higher when fixating an optically degraded stimulus.

This observation suggests that cyclotorsion can be significantly higher when the epithelium is removed during PRK or when the flap is lifted during LASIK.

Becker, ${ }^{9}$ who investigated the influence of body position on dynamic cyclotorsion, could not demonstrate neither a change in static eye position from the sitting to the supine position, which has been reported by several authors ${ }^{2-4}$ using different measuring techniques, nor any difference in dynamic cyclotorsions.

The experimental settings of their experiment are very similar to ours. The differences consist in the distance from the fixation target ( $4 \mathrm{~m}$ in their experiment) and in the use of the eye-lid speculum in our experiment to better simulate a refractive procedure. We have no explanation for the difference between Becker's results and ours, although differences in the experimental settings can have some influence. Previous reports, ${ }^{10}$ with the same apparatus and a similar technique, demonstrated significant differences in eye cyclotorsion from the upright to the supine position in healthy probands.

The influence of body position on cyclotorsion in our study cannot be explained by changes in head position, because the SMI mask provides information on the $x, y$ and $\mathrm{z}$ head position, whereas maximum care was taken in order to keep the head in the same position throughout the experiments. Only a minimum percentage of torsional eye movements $(<1 \%)$ was discarded since they might have been induced by changes in head position. The image quality index was constantly high, assuring that the measure of torsion was correct throughout the experimental settings.

The higher cyclotorsions in the supine position might be due to changes in the vestibular inputs.

As far as the influence of blurring is concerned, previous Authors ${ }^{8}$ could demonstrate a significant worsening of the mean stability of fixation as a function of optical blur on the $x$ and $y$ axes. The visual feedback might play an important role in maintaining torsional stability, as suggested by van Rijn et al, ${ }_{1}^{11}$ who could demonstrate that the stability of cyclovergence is enhanced by the use of a structured visual background. The high range of temporal variability on the torsional plane can be explained by the observation that drifts on the torsional plane do not displace images from the fovea.

A possible limitation of our study is the fact that we had emmetropic subjects fixate a $2 \mathrm{~m}$ distance target. With this experimental setting, we had the advantage of constantly having a clear corneal surface that assured high precision in the assessment of torsional movements. Although a similar setting was used in a previously published paper, ${ }^{9}$ it might be that patients with different refractive errors, fixating a near light, might behave differently as far as torsional eye movements are concerned. Furthermore, it is very difficult to exactly calculate the impact of torsion on ablation results, because this is largely dependent on laser-related variables. The spots' energy, size, rate, as well as the overall design of the ablation profile can influence the final results independently of any torsional movement. Nevertheless, our results compare favourably with those obtained by Gerten ${ }^{12}$ in a real refractive setting. This author could demonstrate a $1.4^{\circ}$ average change after cutting the flap; the torsion evaluation error ranged from 0 to $2^{\circ}$ in $19 \%$ of patients, from $2^{\circ}$ to $5^{\circ}$ in $45 \%$, from $5^{\circ}$ to $10^{\circ}$ in $28 \%$ and was more than $10^{\circ}$ in $9 \%$. Furthermore, Gerten ${ }^{12}$ could demonstrate in astigmatic patients a $10 \%$ improvement in accuracy, having 76 vs $66 \%$ of patients within $0.5 \mathrm{D}$ of the attempted cylindrical correction when cyclotorsional movements were corrected.

Chernyak ${ }^{4}$ reported that $56 \%$ of the eyes experienced more than $2^{\circ}$ of cyclorotation between the Wavescan measurement and the laser surgery and that $22 \%$ of eyes experienced more than $5^{\circ}$ of cyclorotation.

The percentage of eyes with torsional movements exceeding $5^{\circ}$ is higher in our experimental setting. All the patients in the supine position had at least some measurements exceeding $5^{\circ}$ cyclorotation, although in six subjects only the misalignment lasted longer than $1 \mathrm{~s}$.

While doing the experiments, we realized that emotional factors can play an important role in the amount of cyclotorsional movements. Patients who experienced nervousness during the exam produced an increase in eye movements that might have partially affected the results of this study. Several factors can contribute to the torsional eye movements while the final result of the corneal ablation would be dependent on several laser variables. Nevertheless, our results strongly suggest that, due to the high range of cyclotorsional movements that occur in the supine position when fixating a blurred stimulus and due to the temporal characteristics of eye torsions, active 3D tracking could be a significant improvement especially with the correction of high astigmatism or with the aberrometryguided ablations.

\section{References}

1 Guirao A, Williams D, Cox I. Effect of rotation and translation on the expected benefit of an ideal method to correct the eye's higher-order aberration. J Opt Soc Am A 2001; 18: 1003-1015.

2 Tjon-Fo-Sang MJ, de Faber JT, Kingma C, Beekhuis WH. Cyclotorsion: a possible cause of residual astigmatism in refractive surgery. J Cataract Refract Surg 2002; 28: 599-602. 
3 Swami AU, Steinert RF, Osborne WE, White AA. Rotational malposition during laser in situ keratomileusis. Am J Ophthalmol 2002; 133: 561-562.

4 Chernyak DA. Cyclotorsional eye motion occurring between wavefront measurement and refractive surgery. J Cataract Refract Surg 2004; 30(3): 633-638.

5 Graf EW, Maxwell JS, Schor CM. Changes in cyclotorsional and vertical eye alignment during prolonged monocular occlusion. Vis Res 2002; 42: 1185-1194.

6 DiScenna AO, Das V, Zivotofsky AZ, Seidman SH, Leigh RJ. Evaluation of a video tracking device for measurement of horizontal and vertical eye rotations during locomotion. J Neurosci Methods 1995; 58(1-2): 89-94.

7 Teiwes W, Merfeld DM, Young LR. Comparison of the scleral search coil and videoculography techniques for three-dimensional eye movement measurement. In: Fetter M, Haslwanter T, Misslich H, Tweed D, (eds). ThreeDimensional Kinematics of the Eye, Head and Limb Movements.
Overseas Publishers Association: Amsterdam, 1997, pp 429-443.

8 Ukwade MT, Bedell HE. Stability of oculomotor fixation as a function of target contrast and blur. Optom Vis Sci 1993; 70: 123-126.

9 Becker R, Krzizok Th, Wassill H. Use of preoperative assessment of positionally induced cyclotorsion: a videooculographic study. Br J Ophthalmol 2004; 88: 417-421.

10 Gordes RS, Vaseghi S, Pansell T. Influence of the body position to cyclorotation in healthy probands. Ophthalmologie 2002; 99(Suppl 1): 142

11 Van Rijn LJ, Van der Steen J, Collewijn H. Instability of ocular torsion during fixation: cyclovergence is more stable than cycloversion. Vis Res 1994; 34: 1077-1087.

12 Gerten G. Personal communication at the XXI Congress of the ESCRS in Munich 6th-10th September 2003 during his presentation: 'The AK-LASIK: correction of high astigmatism with a new two-steps procedure'. 